Article

Subscriber access provided by Imperial College London | Library

\title{
An analytical pipeline for quantitative characterization of dietary intake: application to assess grape intake
}

Isabel Garcia-Perez, Joram M. Posma, Edward S Chambers, Jeremy K. Nicholson, John C. Mathers, Manfred Beckmann, John Draper, Elaine Holmes, and Gary Frost

J. Agric. Food Chem., Just Accepted Manuscript • DOI: 10.1021/acs.jafc.5b05878 • Publication Date (Web): 24 Feb 2016

Downloaded from http://pubs.acs.org on March 2, 2016

\section{Just Accepted}

"Just Accepted" manuscripts have been peer-reviewed and accepted for publication. They are posted online prior to technical editing, formatting for publication and author proofing. The American Chemical Society provides "Just Accepted" as a free service to the research community to expedite the dissemination of scientific material as soon as possible after acceptance. "Just Accepted" manuscripts appear in full in PDF format accompanied by an HTML abstract. "Just Accepted" manuscripts have been fully peer reviewed, but should not be considered the official version of record. They are accessible to all readers and citable by the Digital Object Identifier (DOI®). "Just Accepted" is an optional service offered to authors. Therefore, the "Just Accepted" Web site may not include all articles that will be published in the journal. After a manuscript is technically edited and formatted, it will be removed from the "Just Accepted" Web site and published as an ASAP article. Note that technical editing may introduce minor changes to the manuscript text and/or graphics which could affect content, and all legal disclaimers and ethical guidelines that apply to the journal pertain. ACS cannot be held responsible for errors or consequences arising from the use of information contained in these "Just Accepted" manuscripts. 
TITLE

An analytical pipeline for quantitative characterization of dietary intake: application to assess grape intake

\section{AUTHORSHIP}

Isabel Garcia-Perez ${ }^{\dagger}$, Joram M. Posma ${ }^{\ddagger}$, Edward S. Chambers ${ }^{\dagger}$, Jeremy K. Nicholson ${ }^{\ddagger}$, John C. Mathers ${ }^{\S}$, Manfred Beckmann", John Draper" ${ }^{\#}$, Elaine Holmes ${ }^{\ddagger}$, Gary Frost ${ }^{\star \dagger}$

\footnotetext{
${ }^{\dagger}$ Nutrition and Dietetic Research Group, Division of Endocrinology and Metabolism, Imperial College London, W12 0NN, UK (IGP, ESC, GF)

${ }^{\ddagger}$ Computational and Systems Medicine, Department of Surgery and Cancer, Imperial College London, SW7 2AZ, UK (JMP, JKN, EH)

§Human Nutrition Research Centre, Institute of Cellular Medicine, Newcastle University, NE4 5PL, UK (JCM)

\#Institute of Biological, Environmental and Rural Sciences, Aberystwyth University, SY23 3DA, UK (MB, JD)

*Phone: +44(0)2075943225. E-mail: g.frost@imperial.ac.uk
} 


\section{Abstract}

2 Lack of accurate dietary assessment in free-living populations requires discovery of

3 new biomarkers reflecting food intake qualitatively and quantitatively to objectively

4 evaluate effects of diet on health. We provide a proof-of-principle for an analytical

5 pipeline to identify quantitative dietary biomarkers. Tartaric acid was identified by

6 Nuclear Magnetic Resonance spectroscopy as dose-responsive urinary biomarker of

7 grape intake and subsequently quantified in volunteers following series of 4-day

8 dietary interventions incorporating $0 \mathrm{~g} /$ day, $50 \mathrm{~g} /$ day, $100 \mathrm{~g} /$ day and $150 \mathrm{~g} /$ day of grapes

9 in standardized diets from a randomized controlled clinical trial. Most accurate 10 quantitative predictions of grape intake were obtained in $24 \mathrm{~h}$ urine samples which

11 have the strongest linear relationship between grape intake and tartaric acid excretion $12 \quad\left(r^{2}=0.90\right)$.

13 This new methodological pipeline for estimating nutritional intake based on coupling

14 dietary intake information and quantified nutritional biomarkers was developed and 15 validated in a controlled dietary intervention study, showing that this approach can 16 improve accuracy of estimating nutritional intakes.

17 Key words: accurate dietary assessment, metabolic profiling, nutritional intake, 18 quantified dietary biomarkers, tartaric acid 
19

20

21

22

23

24

25

26

27

28

29

30

31

32

33

34

35

36

37

38

39

40

41

42

43

\section{INTRODUCTION}

Higher consumption of vegetables and fruits is associated with lower all-cause mortality and recent studies suggest that daily intakes of 5 (1) or 7 or more portions of vegetables and fruits (2) lowers the risk of death, particularly cardiovascular mortality.

Such evidence is the basis for government recommendations to improve eating patterns. However, monitoring compliance to dietary advice at the population level is extremely difficult because existing dietary assessment tools based on self-reporting methods are inherently inaccurate $(3,4)$. Under-reporting was found to be $34 \%$ in men and $33 \%$ in women, with the highest occurrence of under-reporting in obese and overweight individuals $(5,6)$. There is an unmet need for quantifiable dietary biomarkers that accurately reflect consumption of foods and nutrients (4).

Dietary intake biomarkers are based on the concept that excretion levels of foodrelated metabolites are highly correlated with food intake over a given period of time. These biomarkers can be components of the food itself, excreted unchanged or compounds that have undergone metabolic conversion by the human or by the resident gut bacteria. Global metabolic profiling using spectroscopic technology has been applied to detect food-derived compounds (7) including polyphenols (berries) (8), alkyl resorcinols (wheat) (9), proline betaine (citrus fruit) (10), ( $N$-acetyl-)S-methyl-Lcysteine sulfoxide (cruciferous vegetables) (11), and trimethylamine- $\mathrm{N}$-oxide and methylhistidine (oily fish) (8) which are potential biomarkers of original food intake. Metabolic profiling allows an independent and objective assessment of food intake from which energy and nutrients can be calculated. Whilst much of this metabolic profiling research was conducted using panels of metabolites or excretion patterns, few studies have established quantitative relationships between amounts of specific foods consumed and food-derived metabolites excreted. Exceptions include: total 
44 urinary nitrogen for protein intake, urinary potassium and sodium outputs for 45 potassium and sodium intake (12) and proline betaine derived from citrus fruit 46 consumption which exhibits total clearance within 24h (13). However, as with many 47 putative biomarkers of individual foods, proline betaine is not entirely specific to citrus 48 fruits and can be found in low concentrations in alfalfa, pulses, kiwi and pears $(10,14)$.

49 Nevertheless, proline betaine has been shown to be a robust and quantifiable dietary 50 biomarker of citrus intake $(10,13)$.

51 Specific foods may have benefits for specific diseases. For example, consumption of 52 grapes and grape-based products has potential efficacy in cancer prevention (15-18) 53 and is associated with decreased risk for cardiovascular disease (19), however there 54 is no irrefutable evidence for the health benefits of grapes from randomized controlled 55 intervention studies. A methodology for generating reliable markers of specific foods 56 such as grapes would be a valuable addition to the armory of nutritional tools. Here we 57 develop and apply a rapid, accurate and efficient analytical pipeline for assessing food 58 intake using Nuclear Magnetic Resonance (NMR) spectroscopy and demonstrate its 59 application in measuring urinary concentrations of tartaric acid as an indicator of grape 60 intake. 


\section{MATERIALS AND METHODS}

62 Identification of a candidate biomarker for grape intake

63 To explore potential quantifiable biomarkers of intake of grapes, we designed a grape 64 challenge pilot study that was undertaken to characterize the urinary metabolite 65 excretion profile following consumption of grapes. Six volunteers ( 3 men and 3 66 women, age range: 22-32, BMI range: $21.2-25.3 \mathrm{~kg} / \mathrm{m}^{2}$ ) were recruited and 67 participated in a 3-day study. Participants were healthy, non-smokers and did not 68 consume drugs or food supplements regularly. The grapes were administered as part 69 of a standard breakfast consisting of one cup of tea or coffee and a fruit salad 70 containing apple, pineapple and red grapes. The amount of red grapes in the 71 consumed fruit salad was increased over the three consecutive days viz. $50 \mathrm{~g}, 100 \mathrm{~g}$ 72 and $200 \mathrm{~g}$ of red grapes on days 1, 2 and 3, respectively, whilst apple and pineapple 73 intake remained constant. For the $24 \mathrm{~h}$ preceding the grape challenge and throughout 74 the remaining part of each experimental day, participants ate their habitual diet 75 avoiding any products containing grapes, raisins or food products derived from 76 grapes. All urine produced was collected daily into four timed aliquots per day 77 corresponding to $0-4 h, 4-8 h, 8-12 h$ and $12-24 h$ post consumption, using single-use 78 urine containers (International Scientific Supplies Ltd, Bradford, United Kingdom). In 79 addition, a spot urine sample was collected on day 1 prior to consumption of grapes to 80 provide a baseline profile. Urine samples were stored at $-80^{\circ} \mathrm{C}$ until analysis.

\section{Compositional analysis of grapes study}

82 To characterize the chemical composition of varieties of grapes consumed in the UK, 83 ten varieties of red grapes and two varieties of green grapes from five countries were 84 selected according to seasonal availability. Two batches of $400 \mathrm{~g}$ of each variety of 85 grapes were purchased and for each variety $50 \mathrm{~g}$ of grapes were picked from random 
areas of different clusters to provide a representative sample. A total of three replicates of each batch were prepared. Each individual sample was homogenized using a Kenwood KMix Blender for $5 \mathrm{~min}$ and, approximately, $50 \mathrm{ml}$ of must were obtained and then filtered using a stainless steel filter. An aliquot of $1 \mathrm{ml}$ of each sample was centrifuged for $5 \mathrm{~min}$ at $16,000 \mathrm{xg}$, and the supernatant fluid was analyzed by ${ }^{1} \mathrm{H}-\mathrm{NMR}$ spectroscopy. Quantification of the tartaric acid concentration in red and green grapes was carried out using a standard one-dimensional NMR pulse sequence ensuring fully relaxed pre-saturation of the water resonance.

\section{${ }^{1} \mathrm{H}-\mathrm{NMR}$ spectroscopic analysis of urine and grape samples}

An aliquot of each urine sample $(540 \mu \mathrm{l})$ was mixed with $60 \mu$ l of $\mathrm{pH} 7.4$ phosphate buffer containing trimethylsilyl-[2,2,3,3,-2 ${ }^{2}$ 4] - propionate (TSP) as an internal chemical shift reference before being prepared for the NMR spectroscopic analysis following the protocol described by Dona et al. (20). Urine samples were analyzed in 96-well plates containing one quality control (QC) sample every ten samples. QC samples were prepared by pooling $50 \mu$ l of each urine sample. Filtered homogenates $(400 \mu \mathrm{l})$ of representative samples of the grapes given to participants were mixed with $200 \mu$ of $\mathrm{pH} 7.4$ phosphate buffer. QC samples of grape homogenates were prepared by pooling $50 \mu$ l of each grape fluid sample and analyzed every ten samples.

${ }^{1} \mathrm{H}-\mathrm{NMR}$ spectroscopy was performed at $300 \mathrm{~K}$ on a Bruker $600 \mathrm{MHz}$ spectrometer (Bruker BioSpin, Karlsruhe, Germany) using the following standard one-dimensional pulse sequence with saturation of the water resonance: $R D-g z, 1-90^{\circ}-t-90^{\circ}-t_{m}-g z, 2-$ $90^{\circ}-\mathrm{ACQ}$, where $\mathrm{RD}$ is the relaxation delay, $t$ is a short delay typically of about $4 \mu \mathrm{s}$, $90^{\circ}$ represents a $90^{\circ}$ radio frequency (RF) pulse, $t_{m}$ is the mixing time $(10 \mathrm{~ms}), \mathrm{gz}, 1$ and gz,2 are magnetic field z-gradients both applied for $1 \mathrm{~ms}$, and ACQ is the data acquisition period (2.7s). Water suppression was achieved through continuous wave 
111 irradiation at the water resonance frequency using $25 \mathrm{~Hz} \mathrm{RF}$ strength during RD and 112 also during $t_{m}$. The receiver gain was set to 90.5 for all experiments. Each urine 113 spectrum was acquired using 4 dummy scans, 32 scans, $64 \mathrm{~K}$ time domain points and 114 with a spectral window set of $20 \mathrm{ppm}$. Prior to Fourier transformation, the free 115 induction decays were multiplied by an exponential function corresponding to a line 116 broadening of $0.3 \mathrm{~Hz}$. To achieve accurate quantification of metabolites, it was 117 necessary to ensure that both tartaric acid and TSP resonances were fully relaxed 118 using a long RD $\left(7 \times t_{1}\right)$ between each pulse. The inter-pulse delay time $d_{1}$ was 119 therefore set to $100 \mathrm{~s}(21)$.

120 Pre-processing of NMR spectra

121 The ${ }^{1} \mathrm{H}-\mathrm{NMR}$ spectra were digitized over the range of $\delta 10.0$ to -0.5 and imported into 122 MATLAB (2014a, Mathworks Inc., USA), and automatically phase- and baseline123 corrected. Urine spectra were then referenced to the internal standard, TSP at $\delta 0.0$. 124 The spectral regions occupied by water and urea $(\delta 4.45-6.95)$ and TSP $(<\delta 0.35)$ were 125 excluded. Each spectrum was normalized to the total urine volume excreted in order 126 to correct differences in urinary dilution. Spot urine samples were normalized to the 127 spot volume. Each spectrum of grape juice extract was phased and baseline corrected 128 as above, and normalized to the TSP signal.

\section{Statistical analysis}

130 Urinary global profiling analysis in combination with unsupervised Principal 131 Component Analysis (PCA) (22) and supervised Partial Least Squares Discriminant 132 Analysis (PLS-DA) (23) was applied to identify candidate biomarkers of grape intake. 133 PCA was used to visualize any trends in the data and these trends were then further 134 analyzed using PLS-DA and Monte-Carlo cross-validation (MCCV) (24). For each 135 component in the PCA model the percentage of explained variance $\left(R^{2} x\right)$ was 
136 calculated. The variability of the predicted scores was visualized using Kernel Density

137 Estimation (KDE). Across all MCCV models, the mean score and variance of the score

138 for each sample were used to calculate the KDE. Specifically, for each sample the 139 mean and standard deviation across all MCCV models can be used to give a normal 140 distribution of the predicted scores. Taking the sum of all individual distributions within 141 each class then yields the KDE as shown in the figures. The stability (variance) of 142 each regression coefficient was assessed across the MCCV models using bootstrap 143 resampling (25) of each model. Using the variance and mean regression coefficient, a 144 t-score, and subsequently a $P$-value, were calculated. The $P$-values were corrected for 145 multiple testing using the Storey-Tibshirani (26) False Discovery Rate (q-value). The 146 goodness of fit $\left(R^{2} \mathrm{Y}\right)$ of the MCCV models was calculated across all models using the 147 training data and the goodness of prediction $\left(Q^{2} Y\right)$ for the test data.

148 Hierarchical cluster analysis (HCA) was used to investigate correlations among the 149 identified biochemical components of different variaties of grapes, specifically to 150 determine whether the concentration of tartaric acid shows similarity with other 151 compounds. To quantify the relationship, HCA was used to determine clustering in the 152 data. Significance of the correlation between pairs of compounds was assessed based 153 on a Bonferroni correction to the P-values. HCA was applied to the resulting 154 correlation matrix and the optimal number of clusters was determined by calculating 155 the modularity (27) of the network and choosing the highest modularity as optimal 156 number of clusters. The modularity is a weighted measure between the number of 157 links (correlations) within a cluster and the number of links from one cluster to other 158 clusters, with clusters defined by cutting at different heights of the hierarchical 159 clustering tree. The highest modularity indicates that, relatively, there are more within160 cluster links compared to between-cluster links. 
161 Standard linear regression was used to relate grape intake to tartaric acid excretion.

162 The variability of the regression coefficient is visualized in corresponding plots as the $16395 \%$ confidence intervals $(\mathrm{Cl})$ estimate by bootstrap resampling of the regression 164 coefficient. The squared correlation coefficient of the model $\left(r^{2}\right)$ is a measure of 165 linearity of the data and corresponding model. Bland-Altman (28) plots were used to 166 show the agreement between the estimated intake of grapes versus the real intake of 167 grapes.

168 PCA and PLS-DA analyses were also carried out on spectral data of grape 169 homogenates normalized to volume in MATLAB to compare different grape varieties.

170 Correlation of spectral variables using Statistical Total Correlation Spectroscopy (29)

171 and Subset Optimization by Reference Matching (30) were used as data-driven 172 approaches to aid metabolite identification. Confirmation of metabolite identities was 173 obtained using 1D and 2D NMR experiments (spiking of chemical standards, J174 Resolved spectroscopy, Total Correlation Spectroscopy, Hetero-nuclear Single 175 Quantum Coherence spectroscopy).

\section{Quantification of tartaric acid related to grape intake}

177 Nineteen volunteers (10 male and 9 female, age range: 25-60, BMI range: 21.1$17833.3 \mathrm{~kg} / \mathrm{m}^{2}$ ) attended the NIHR/Wellcome Trust Imperial CRF for four 4-day inpatient 179 periods separated by a period of $>3$ days. Potential subjects were excluded if they had 180 clinically significant illnesses, were taking prescription medication, current smokers, a 181 history of substance abuse, and any abnormalities detected on physical examination, 182 electrocardiography, or screening blood tests (measurement of complete blood count, 183 electrolytes, fasting glucose and lipids, thyroid function and liver function). Women 184 were ineligible if they were pregnant or breast-feeding. In a random order, participants 185 were provided with four different diets throughout each of the 4-day inpatient periods. 
Each of the four diets represented 25\% (diet 1), 50\% (diet 2), 75\% (diet 3) and 100\% (diet 4) of the healthy eating targets based on UK recommendation for fruits, fats, sugars, vegetables, carbohydrates, fiber and salt. Grapes were consumed as an afternoon snack two hours after lunch. Alcohol and grape derived products were not provided as part of any of the four diets.

The amounts of red grapes administered daily were $0 \mathrm{~g}$ (diet 1), $50 \mathrm{~g}$ (diet 2), $100 \mathrm{~g}$ (diet 3) and $150 \mathrm{~g}$ (diet 4) (Figure 1). The randomization procedure was conducted by an investigator not directly involved in the study with the use of opaque, sealed, sequentially numbered envelopes that each contained a random order for the four dietary interventions. The envelopes were stored securely and opened in sequence by an investigator (ESC) once volunteers had been recruited. Volunteers and investigators could not be blinded during data collection; however, all investigators conducting data analysis were blinded to the randomization order. Different varieties of red grapes from different countries were provided according to seasonal availability. Wine, raisins and any fruit juice were excluded from the experimental diets. Moreover, volunteers did not take any supplements and minimal physical activity was imposed. Fasting spot urine samples were collected on arrival at the NIHR/Wellcome Trust Imperial Clinical Research Facility and daily thereafter for the four days of each of four dietary interventions. Each participant collected cumulative urine samples (CS) daily, over the four-day period of each dietary intervention, from after breakfast to before lunch (CS1), from after lunch to before dinner (CS2) and from after dinner to next day fasting urine sample (CS3). Finally, a 24h urine sample was obtained by pooling CS1, CS2 and CS3. In addition, a spot sample was collected daily two hours after the afternoon snack. Aliquots of urine were transferred into Eppendorf tubes and stored at $-80^{\circ} \mathrm{C}$ until analysis by ${ }^{1} \mathrm{H}-\mathrm{NMR}$ spectroscopy. All subjects provided informed, written 
211 consent prior to the clinical trial (Registration No: ISRCTN 43087333), which was

212 approved by the London Brent Research Ethics Committee (13/LO/0078). All studies

213 were carried out in accordance with the Declaration of Helsinki.

214 Monitoring in-patient volunteers for $\mathbf{2 4 h}$ tartaric acid excretion

215 Samples from day 3 of each dietary intervention were chosen to monitor volunteers'

216 excretion (Figure 1) of tartaric acid over $24 \mathrm{~h}$, as well as in the fasting urine sample of

217 day 3 of the study, spot urine sample collected $2 \mathrm{~h}$ after grapes consumption and the

218 fasting urine sample on the day 4 of the study.

219 Tartaric acid gives rise to a single peak in the NMR spectrum at $\delta 4.34$. This signal was 220 integrated for the urine samples using an automated algorithm (31). The amount of

221 tartaric acid excreted in $24 \mathrm{~h}$ was calculated by dividing the corresponding integral by

222 the number of ${ }^{1} \mathrm{H}$ of tartaric acid signals $\left(4 \times{ }^{1} \mathrm{H}\right)$ and multiply with the number of ${ }^{1} \mathrm{H}$ in

223 TSP $\left(9 x^{1} H\right)$. Then multiplying with the concentration of TSP in the sample gives the

224 concentration of tartaric acid. Three calibrations curves corresponding to CS2, CS3

225 and $24 \mathrm{~h}$ urine samples, were built to establish the relationship between excretion of

226 tartaric acid and the amount of grapes consumed.

227 Prediction of grape intake based on tartaric acid urinary excretion in a 228 randomized highly controlled clinical trial

229 Tartaric acid was quantified for each of the spot and cumulative sample sets 230 (corresponding to $0-3 \mathrm{~h}$ post consumption (CS2); $3-15 \mathrm{~h}$ post consumption (CS3) and 231 the $24 \mathrm{~h}$ urine cumulative samples) for each of the four levels of grape intake $(0,50$, $232100,150 \mathrm{~g})$ to investigate how accurately grape intake could be estimated in a highly 233 controlled clinical trial. The models were trained using calibration curves built using the 234 tartaric acid signals from urine spectra derived from samples obtained on the third day 235 of the study $(n=304)$. The model was then used to predict the quantity of grape intake 
236 using samples collected on the first and the second day of each dietary intervention $237 \quad(n=608)$. 
238

239

240

241

242

243

244

245

246

247

248

249

250

251

252 the $12-24 \mathrm{~h}$ collection.

\section{$253 \quad{ }^{1} \mathrm{H}-\mathrm{NMR}$ global profiling of red and green grapes}

254 To confirm the dietary origin of tartaric acid we obtained $96{ }^{1} \mathrm{H}-\mathrm{NMR}$ global profiles of 255

\section{RESULTS AND DISCUSSION}

\section{Monitoring ${ }^{1} \mathrm{H}-\mathrm{NMR}$ urinary global profile over $24 \mathrm{~h}$ following grape intake}

The tartaric acid signal corresponding to a singlet at $\delta 4.34$ was identified from the global profiling analysis of the pilot human intervention study $(n=6)$ as a candidate marker of grape consumption. The signal for tartaric acid was absent in the baseline urine sample prior to grape consumption and showed an incremental increase in intensity as the amount of grapes consumed increased. Although other signals from metabolites such as glucose, hippurate and 4-hydroxyhippurate were also qualitatively associated with grape intake, the tartaric acid signal was the only peak in the ${ }^{1} \mathrm{H}-\mathrm{NMR}$ global metabolic profile observed to increase proportionally with incremental grape intake (Figure 2A) over the three consecutive days in all participants. The urinary excretion kinetics of tartaric acid was calculated. In all participants, excretion of tartaric acid peaked between $4 \mathrm{~h}$ and $8 \mathrm{~h}$ post-intervention and the majority of the excretion occurred in the first $12 \mathrm{~h}$. Tartaric acid concentrations declined almost to baseline after red $(n=63)$ and green grapes $(n=33)$ from different varieties and countries (as listed in Figure 2B). As expected, tartaric acid was one of the dominant compounds from the 31 metabolites identified in the global metabolic profiles of grapes (Supplementary Figure 1). The PCA scores plot showed clustering of grapes according to variety and country (Figure 2B) with the Black Princess (Chile) and the Sharad (India) varieties being most distinctive in profile. Moreover, the PCA model including all the red and green grape samples showed a trend in clustering according to the color of the grape (Figure 2C). Systematic differences between red and green grapes were determined 
263 from the PLS-DA model $\left(R^{2}=0.65, Q^{2}{ }^{2}=0.52\right)$ (Figure 3A, B), including significantly

264 higher concentrations of phenylalanine and leucine in green grapes whereas ethyl 265 glucuronide was significantly higher in the red grapes (Figure 3C). The concentration 266 of tartaric acid (Figure 3C, inset) was not significantly different between red and green 267 grapes or varieties indicating that it could have general applicability as a marker of 268 grape intake.

269 Correlations between the 31 small molecules displayed in a heat-map (Supplementary 270 Figure 1) showed grouping of chemical components of the grape homogenate. 271 Examples of distinct clusters, correspond to amino acids in one and for instance 272 ethanol, acetoin and 2,3-butanediol (markers of fermentation) in another cluster. It 273 shows that tartaric acid is an independent component in grapes as it is independent of 274 other small molecules found in grapes.

275 Assessment of tartaric acid as a quantitative biomarker of grape intake in 276 human urine samples

277 As expected, the third and fourth day spot-fasting urine samples and the CS1 urine 278 sample (cumulative sample from after breakfast to before lunch) collected during the 279 controlled clinical trial did not contain any tartaric acid as the samples were collected 280 before volunteers ingested red grapes (afternoon snack).

281 The calibration curves for tartaric acid calculated using urine samples obtained on the 282 third day of each dietary intervention showed a linear relationship between quantity of 283 grapes consumed and tartaric acid excreted, with the exception of the spot urine 284 samples. Although, spot urine samples collected $2 \mathrm{~h}$ after grape intake showed some 285 evidence of tartaric acid excretion, there were no significant differences in 286 concentrations (Figure 4A) in relation to the different amounts of grapes consumed $287\left(r^{2}=0.04\right)$. The CS2 urine samples (collected from after lunch to before dinner) (Figure 
288 4B), which contained the urine excreted in the first three hours following consumption 289 of grapes as an afternoon snack, showed a linear relationship with a correlation 290 coefficient of $r^{2}=0.58$.

291 The correlation coefficient defining the relationship between amount of grape intake 292 and tartaric acid excretion corresponding to samples that were collected overnight 293 (CS3: 12h collection from after dinner to next day fasting urine sample) was stronger $294\left(r^{2}=0.80\right)$ than the correlation coefficient (Figure 4C) found for the previous period 295 (CS2). However, the 24h calibration curve (Figure 4D), showed the highest correlation 296 coefficient $r^{2}=0.90$ and therefore it was used to quantify the total $24 \mathrm{~h}$ urinary tartaric 297 acid excretion for day three. On average, $0.16,0.30$ and $0.49 \mathrm{mMol}$ of tartaric acid was 298 excreted in $24 \mathrm{~h}$ urine samples after eating 50,100 and $150 \mathrm{~g}$ of grapes, respectively. 299 The mean, standard deviation and 95\% confidence interval of urinary tartaric acid 300 excreted calculated for the three urine collection period: $24 \mathrm{~h}(0.161 \pm 0.035 \mathrm{mMol}$ 301 (0.101-0.231)), CS3 $(0.115 \pm 0.040 \mathrm{mMol}(0.026-0.195))$ and CS2 $(0.046 \pm 0.023 \mathrm{mMol}$ 302 (0.014-0.093)) samples after eating $50 \mathrm{~g}$ of grapes.

303 Calculation of the ratio of tartaric acid consumed and tartaric acid excreted in 304 the urine

305 The mean concentration of tartaric acid in $50 \mathrm{~g}$ of red grapes (ten different varieties) 306 and in $50 \mathrm{~g}$ of green grapes (two different varieties) was $0.84 \pm 0.03 \mathrm{mMol}$ for red and $3070.85 \pm 0.08 \mathrm{mMol}$ for green grapes (Supplementary Table 1). These results 308 corroborated the PLS-DA results, which found no significant differences in the quantity 309 of tartaric acid according to grape color or variety. The amount of tartaric acid excreted 310 unchanged in the urine after consumption of $50 \mathrm{~g}$ of red grapes represented $19.2 \%$ of 311 the amount consumed. Of this $5.5 \%$ was excreted in the first $3 \mathrm{~h}$ post consumption 312 (CS2) and the remaining $13.7 \%$ was excreted $3-15$ h post consumption (CS3). 
313 Estimation of grape intake based on tartaric acid urinary excretion in a 314 randomized highly controlled clinical trial

315 Finally, we assessed the quantity of grapes consumed during the controlled clinical 316 trial based on the models calculated from tartaric acid excretion in the urine samples 317 collected on day 3 . The CS2, CS3 and $24 \mathrm{~h}$ urine samples collected during the first and 318 the second day of the control clinical trial were used as a test set of samples for this 319 purpose. The amount of urinary tartaric acid excreted was interpolated from the 320 calibration curve built with the CS2, CS3 and $24 \mathrm{~h}$ urine samples obtained on the third 321 day of the study in order to estimate the amount of grapes consumed by the 322 volunteers on the first two days in each intervention.

323 The Bland-Altman plots of the actual and estimated grape intake in CS2, CS3 and 24h 324 samples are shown in Figure 5A, B and C, respectively. These plots highlight that the 325 prediction of mean grape intakes in grams were close to the known intakes of $50 \mathrm{~g}$ $326(50.9 \mathrm{~g}), 100 \mathrm{~g}(91.4 \mathrm{~g})$ and $150 \mathrm{~g}(163.0 \mathrm{~g})$ of grapes and also verify that tartaric acid 327 was not present in diet 1 where grapes were not consumed. We found that accuracy 328 of the predicted intake was inversely proportional to the amount of grapes consumed 329 for all collection periods (CS2, CS3 and 24h). In general, the most accurate prediction 330 of intake was found in the $24 \mathrm{~h}$ urine sample (Figure 5 ) indicating that collection of $24 \mathrm{~h}$ 331 samples is the most appropriate strategy for estimation of grape intake. 
332

333

334

335

336

337

338

339

340

341

342

343

344

345

346

347

348

349

350

351

352

353

354

355

356

\section{Development of a quantifiable biomarker for grape consumption}

There is a need to develop quantifiable dietary biomarkers for a range of food and nutrients to identify healthy and/or unhealthy eating patterns, since examples of validated quantifiable nutritional biomarkers are rare. Development of measurable biomarkers of intake of specific foods in body biofluids presents a complex challenge and is a multistage procedure involving: i) discovery and identification of chemical(s) reflecting exposure to specific dietary components, ii) assignment of candidate biomarkers to endogenous or exogenous origin, iii) validation of candidate biomarker in an independent cohort, iv) evaluation of the most appropriate biological sample for quantification of the biomarker and $v$ ) investigation of the specificity and sensitivity of the proposed nutritional biomarker with respect to estimating exposure to specific food or food groups in an epidemiologic context.

In the current study, we developed a quantifiable biomarker for grape intake. ${ }^{1} \mathrm{H}-\mathrm{NMR}$ spectra of urine specimens from a pilot study showed tartaric acid to be a candidate biomarker reflecting exposure to incremental grape consumption. The follow-up kinetic study showed that the majority of tartaric acid was excreted between the first $4 \mathrm{~h}$ and 8h post-intervention in all participants, with almost complete excretion within $24 \mathrm{~h}$ postconsumption (Figure 2A). The dietary origin of tartaric acid as natural organic acid in grapes at high concentrations is well known. It is also present in lower amounts in bananas, cranberries and tamarinds, but rare in most other common plants $(32,33)$. Traces of tartaric acid can also be found in processed foods as an acidifying agent (34). PCA analysis of red grapes showed clustering according to variety and country (Figure 2B), which has been demonstrated due to soil, climatic region, and cultivar practices (35) and is reflected in the variation of sugars, amino acids and organic acids. However, differences in chemical composition reflected in PCA and PLS-DA 
357 models were unrelated to tartaric acid excretion. Tartaric acid was found in all varieties

358 of grapes tested, and the concentration was similar between varieties (Supplementary

359 Table 1). Thus it is feasible to suggest that tartaric acid serves as a robust, NMR-

360 quantifiable biomarker of grape intake, independent of origin and grape variety.

361 Evaluation of optimal sampling strategy

362 We evaluated the most appropriate sampling strategy for detection and quantification

363 of dietary biomarkers based on $24 \mathrm{~h}$ total urinary biomarker excretion vs. cumulative

364 timed and spot urine collections. The evaluation was conducted by monitoring $24 \mathrm{~h}$

365 urinary excretion of tartaric acid in volunteers attending a highly controlled dietary

366 intervention study. Findings unequivocally indicated that the cumulative $24 \mathrm{~h}$ collection

367 performed best in terms of the ability to accurately estimate the quantity of grapes

368 consumed and to detect and quantify tartaric acid. Evaluation of spot urine samples,

369 as a more practical alternative in terms of study cost and participant compliance,

370 indicated that it was not possible to obtain accurate estimates of grape consumption

371 from spot urines obtained either at 7 am following an overnight fast or $2 \mathrm{~h}$ after grape

372 intake. Although there was evidence of urinary excretion $2 \mathrm{~h}$ after grape intake in the

373 spot urine samples, there was no strong association between dose and amount of

374 tartaric acid excreted. This can most likely be explained by inter- and intra-individual

375 variability in human metabolism. Tartaric acid is an exogenous compound, the majority

376 of which is not absorbed. Our results concur with previous human metabolism studies

377 suggesting that $15-20 \%$ of the dietary tartaric acid is excreted in the urine unchanged

$378(36,37)$. Tartrate either undergoes bacterial digestion in the large intestine, by at least

37923 varieties of bacteria, or it is excreted in the stool in the form of an insoluble salt

380 such as calcium tartrate (36), which can affect the ratio of tartaric acid excreted in

381 feces vs. urine and explain inter-individual differences in excretion. 
382 However, cumulative urine samples CS2 and CS3 showed a linear relationship 383 between grape intake and tartaric acid urinary excretion. These results emphasize the 384 importance of understanding the kinetics of biomarker excretion in order to select the 385 386 best time and sampling strategy, particularly if spot urine samples are to be used to detect specific dietary biomarkers. The optimal sampling time should correspond to the peak excretion time, which will be specific for each dietary biomarker. For example, proline betaine is predominantly detected between 2-6h after citrus intake (10), whilst the optimal window for tartaric acid was between 4-8h after grape intake. With this in mind, nutritional epidemiological studies applying global metabolic profiling strategies should collect the first spot urine sample in a 3-6h time window after the meal or food challenge. It is crucial that urine is collected at the same time for each study participant to avoid introduction of unnecessary variation. Consideration should be given as to whether the biomarker is unique to a given food: for example tartaric acid is itself added as a preservative to some processed foods and beverages. Moreover, it is important to note that sample preservatives can react with biomarkers an example being the complex tartaric acid forms with boric acid (38) altering the intensity and shape of the peak. As expected, $24 \mathrm{~h}$ urine samples remain the best option for total quantification of tartaric acid as a dietary biomarker since total excretion occurs in this time period regardless of inter-individual differences.

\section{Limitations in the proposed strategy for the quantification of dietary biomarkers}

The major confounder for assigning individual chemicals as biomarkers of specific foods or nutrients is the fact that these candidate biomarkers are seldom unique to a specific food. Urinary tartaric acid, in addition to being a major component of grapes, is also found in high concentrations in grape juice and wine, whilst lower concentrations can be found in other fruits or as additives. We found that $19.2 \%$ of all 
407 tartaric acid present in $50 \mathrm{~g}$ of grapes is excreted in the urine unchanged, therefore 408 over $80 \%$ of tartaric acid is metabolized to other compounds. However, to address the 409 lack of specificity of biomarkers, one solution to for instance differentiate grape and 410 wine-derived tartaric acid is to utilize biomarker patterns to ascertain dietary origin.

411 Biomarkers of wine intake identified from interventional studies include gallic acid, 4-o412 methyl gallic acid (39), caffeic acid and catechins. Of these, resveratrol is the only 413 wine intake biomarker validated in clinical and epidemiological studies (40, 41). 414 Therefore, resveratrol could be used to estimate the amount of tartaric acid expected 415 to derive directly from wine, and will allow the differentiation of urinary tartaric acid 416 excreted as a consequence of grape intake or wine intake or both. We found the 417 amount of tartaric acid to be comparable between red and white grapes, therefore it is 418 a stable biomarker for grapes. However, it has been shown that resveratrol is found in 419 higher concentrations in red and white grapes and has been validated as biomarker of 420 red wine (34). In the case of intake of both red grapes and red wine, assessing the 421 ratio between resveratrol and tartaric acid excreted in urine following wine intake alone 422 is needed in order to determine the amount tartaric acid that comes from wine and 423 grapes. Ethanol and ethylglucuronide can also be used to assess consumption of 424 alcoholic beverages in general to supplement using resveratrol alone.

425 Despite the fact that many studies have focused on the identification of wine $(40,41)$ 426 or grape juice (42) biomarkers, we are not aware of any studies reporting 427 (quantitative) biomarkers of grape intake to date. The present work applies a new 428 analytical pipeline to assess tartaric acid as a quantitative biomarker of grape intake 429 that could be used in clinical and epidemiological studies in order to assess accurate 430 grape intake. Grapes are rich in polyphenols, which contain antioxidants, conferring 431 health benefits such as reduced risk of certain cancers. Dietary health policies are 
432 based on traditional dietary assessment methods, which are prone to misreporting. 433 We propose a strategy using NMR as an alternative to current dietary reporting 434 methods and demonstrate its applicability in a controlled nutritional trial. However this 435 strategy can be extendable to other analytical techniques in order to quantify other 436 types of compounds.

437 In summary, we developed an analytical pipeline employing multivariate statistics for 438 identifying a candidate food intake biomarker, followed by calculation of urinary 439 concentration from ${ }^{1} \mathrm{H}-\mathrm{NMR}$ signal intensities and estimating grape intake using 440 calibration curves. We describe a new dietary assessment tool that can be used to 441 confirm intake of specific foods. We exemplified this approach using tartaric acid as a 442 quantifiable biomarker of grape intake. Although this strategy was developed in the 443 context of a highly controlled dietary intervention study, we provide proof-of-principle 444 that coupling self-recorded dietary intake information with quantified nutritional 445 biomarkers may be used to achieve a more objective measure of dietary exposure. 446 Joining efforts from the nutritional and epidemiological researches identifying as many 447 quantitative dietary biomarkers as possible will allow us in future to accurately assess 448 dietary intake both individually and in a population framework. Further studies are 449 required to validate the application of this strategy to assess accurate dietary intake in 450 free-living people.

\section{ABREVIATIONS}

452 BMI: Body Mass Index; $\mathrm{Cl}$ : Confidence Interval; CS: Cumulative (urine) Sample; ${ }^{1} \mathrm{H}$ : 453 proton; HCA: Hierarchical Cluster Analysis; KDE: Kernel Density Estimation; MCCV: 454 Monte-Carlo Cross Validation; NMR: Nuclear Magnetic Resonance; PCA: Principal 455 Component Analysis; PLS-DA: Partial Least Squares Discriminant Analysis; QC: 456 Quality Control; $Q^{2}$ : goodness of prediction; $R^{2}{ }_{Y}$ : goodness of fit; $r^{2}$ : squared 
457 correlation coefficient; RD: Relaxation Delay; RF: Radio-Frequency; $t_{m}$ : mixing time;

458 TSP: trimethylsilyl-[2,2,3,3,- $\left.{ }^{2} \mathrm{H}_{4}\right]$-propionate.

459 ACKNOWLEDGMENTS

460 Kevin Walsh and Ivan Dexeus for their support during the clinical trial.

461 SUPPORTING INFORMATION

462 Supplementary Figure 1: Correlation of 31 identified compounds in ${ }^{1} \mathrm{H}-\mathrm{NMR}$ global 463 profiles of red and green grapes visualized in a heat map with hierarchical clustering 464 applied.

465 Supplementary Table 1: Mean values of quantified tartaric acid in different varieties of 466 red and green grapes from different countries.

467 AUTHOR INFORMATION

468 Corresponding author

469 *Phone: +44(0)2075943225. E-mail: g.frost@imperial.ac.uk

470 Author contributions

471 IGP, JMP, EH and GF designed research; IGP and ESC conducted the clinical trial; 472 IGP conducted research; JMP and IGP analyzed data and performed statistical 473 analyses; IGP, JMP, JKN, JCM, JD, EH and GF wrote the paper. GF had final 474 responsibility for final content. All authors read and approved the final manuscript.

475 Funding

476 IGP is supported by an NIHR postgraduate research fellowship, GF is supported by an 477 NIHR senior investigator award. JCM, JD, MB, GF and EH are supported by an MRC 478 grant entitled Metabolomics for Monitoring Dietary Exposure (Ref: MR/J010308/1).

479 This study was supported by the Wellcome Trust NIHR Clinical Research Facility. The 480 Section of Investigative Medicine is funded by grants from the MRC, BBSRC, NIHR, 481 and an Integrative Mammalian Biology (IMB) Capacity Building Award. 
482 Notes

483 GF has personal links with the food industry through Unilever, Nestlé and Malaysian 484 Palm Oil Board. No other authors declare a conflict of interest. 


\section{REFERENCES}

485 1. Wang, X.; Ouyang, Y.; Liu, J.; Zhu, M.; Zhao, G.; Bao, W.; Hu, F. B., Fruit and vegetable 486 consumption and mortality from all causes, cardiovascular disease, and cancer: systematic review and 487 dose-response meta-analysis of prospective cohort studies. BMJ 2014, 349, g4490.

$488 \quad 2 . \quad$ Oyebode, O.; Gordon-Dseagu, V.; Walker, A.; Mindell, J. S., Fruit and vegetable consumption 489 and all-cause, cancer and CVD mortality: analysis of Health Survey for England data. Journal of 490 epidemiology and community health 2014, 68, 856-62.

$491 \quad 3 . \quad$ Olendzki, B. C.; Ma, Y.; Hebert, J. R.; Pagoto, S. L.; Merriam, P. A.; Rosal, M. C.; Ockene, I. S., 492 Underreporting of energy intake and associated factors in a Latino population at risk of developing 493 type 2 diabetes. J Am Diet Assoc 2008, 108, 1003-8.

494 4. Ismail, N. A.; Posma, J. M.; Frost, G.; Holmes, E.; Garcia-Perez, I., The role of metabonomics as 495 a tool for augmenting nutritional information in epidemiological studies. Electrophoresis 2013, 34, 496 2776-86.

$497 \quad 5 . \quad$ Kelly, M. T.; Rennie, K. L.; Wallace, J. M.; Robson, P. J.; Welch, R. W.; Hannon-Fletcher, M. P.; 498 Livingstone, M. B., Associations between the portion sizes of food groups consumed and measures of 499 adiposity in the British National Diet and Nutrition Survey. The British journal of nutrition 2009, 101, 500 1413-20.

501 6. Shaneshin, M.; Rashidkhani, B.; Rabiei, S., Accuracy of energy intake reporting: comparison of 502 energy intake and resting metabolic rate and their relation to anthropometric and sociodemographic factors among Iranian women. Archives of Iranian medicine 2012, 15, 681-7.

7. Belton, P. S.; Delgadillo, I.; Holmes, E.; Nicholls, A.; Nicholson, J. K.; Spraul, M., Use of HighField 1 H NMR Spectroscopy for the Analysis of Liquid Foods. J Agr Food Chem 1996, 44, 1483-1487.

8. Lloyd, A. J.; Fave, G.; Beckmann, M.; Lin, W. C.; Tailliart, K.; Xie, L.; Mathers, J. C.; Draper, J., Use of mass spectrometry fingerprinting to identify urinary metabolites after consumption of specific foods. Am J Clin Nutr 2011, 94, 981-991.

9. Marklund, M.; Landberg, R.; Aman, P.; Kamal-Eldin, A., Determination of alkylresorcinol metabolites in human urine by gas chromatography-mass spectrometry. Journal of chromatography. $B$, Analytical technologies in the biomedical and life sciences 2010, 878, 888-94.

10. Heinzmann, S. S.; Brown, I. J.; Chan, Q.; Bictash, M.; Dumas, M. E.; Kochhar, S.; Stamler, J.; Holmes, E.; Elliott, P.; Nicholson, J. K., Metabolic profiling strategy for discovery of nutritional biomarkers: proline betaine as a marker of citrus consumption. Am J Clin Nutr 2010, 92, 436-443.

11. Edmands, W. M. B.; Beckonert, O. P.; Stella, C.; Campbell, A.; Lake, B. G.; Lindon, J. C.; Holmes, E.; Gooderham, N. J., Identification of Human Urinary Biomarkers of Cruciferous Vegetable Consumption by Metabonomic Profiling. J Proteome Res 2011, 10, 4513-4521.

12. Bingham, S. A., Biomarkers in nutritional epidemiology. Public health nutrition 2002, 5, 821-7.

13. Lloyd, A. J.; Beckmann, M.; Fave, G.; Mathers, J. C.; Draper, J., Proline betaine and its biotransformation products in fasting urine samples are potential biomarkers of habitual citrus fruit consumption. The British journal of nutrition 2011, 106, 812-24.

14. Wood, K. V.; Stringham, K. J.; Smith, D. L.; Volenec, J. J.; Hendershot, K. L.; Jackson, K. A.; Rich, P. J.; Yang, W. J.; Rhodes, D., Betaines of alfalfa : characterization by fast atom bombardment and desorption chemical ionization mass spectrometry. Plant physiology 1991, 96, 892-7.

15. Hertog, M. G.; Kromhout, D.; Aravanis, C.; Blackburn, H.; Buzina, R.; Fidanza, F.; Giampaoli, S.; Jansen, A.; Menotti, A.; Nedeljkovic, S.; et al., Flavonoid intake and long-term risk of coronary heart disease and cancer in the seven countries study. Arch Intern Med 1995, 155, 381-6.

16. Shirataki, Y.; Kawase, M.; Saito, S.; Kurihara, T.; Tanaka, W.; Satoh, K.; Sakagami, H.; Motohashi, N., Selective cytotoxic activity of grape peel and seed extracts against oral tumor cell lines. Anticancer Res 2000, 20, 423-6.

$531 \quad 17 . \quad$ Do, M. H.; Lee, S. S.; Kim, J. Y.; Jung, P. J.; Lee, M. H., Fruits, vegetables, soy foods and breast 532 cancer in pre- and postmenopausal Korean women: a case-control study. International journal for 533 vitamin and nutrition research. Internationale Zeitschrift fur Vitamin- und Ernahrungsforschung. 534 Journal international de vitaminologie et de nutrition 2007, 77, 130-41. 
18. Kaliora, A. C.; Kountouri, A. M.; Karathanos, V. T.; Koumbi, L.; Papadopoulos, N. G.; Andrikopoulos, N. K., Effect of Greek raisins (Vitis vinifera L.) from different origins on gastric cancer cell growth. Nutrition and cancer 2008, 60, 792-9.

19. Dohadwala, M. M.; Vita, J. A., Grapes and cardiovascular disease. The Journal of nutrition 2009, 139, 1788S-93S.

20. Dona, A. C.; Jimenez, B.; Schafer, H.; Humpfer, E.; Spraul, M.; Lewis, M. R.; Pearce, J. T.; Holmes, E.; Lindon, J. C.; Nicholson, J. K., Precision high-throughput proton NMR spectroscopy of human urine, serum, and plasma for large-scale metabolic phenotyping. Analytical chemistry 2014, 86, 9887-94.

21. Yang, L.; Ru, G. Y.; Tang, H. R.; Liu, C. Y., Human Urinary Metabolite Quantification in Nuclear Magnetic Resonance-based Metabonomics Using Electronic Internal Reference. Chinese J Anal Chem 2010, 38, 789-794.

22. Hotelling, H., Analysis of a complex of statistical variables into principal components. Journal of Educational Psychology 1933, 24, 417-441.

23. De Jong, S., Simpls - an Alternative Approach to Partial Least-Squares Regression. Chemometr Intell Lab 1993, 18, 251-263.

24. Xu, Q. S.; Liang, Y. Z., Monte Carlo cross validation. Chemometr Intell Lab 2001, 56, 1-11.

25. Efron, B., Nonparametric Estimates of Standard Error - the Jackknife, the Bootstrap and Other Methods. Biometrika 1981, 68, 589-599.

26. Storey, J. D.; Tibshirani, R., Statistical significance for genomewide studies. Proc Natl Acad Sci U S A 2003, 100, 9440-5.

27. Guimera, R.; Nunes Amaral, L. A., Functional cartography of complex metabolic networks. Nature 2005, 433, 895-900.

28. Bland, J. M.; Altman, D. G., Statistical methods for assessing agreement between two methods of clinical measurement. Lancet 1986, 1, 307-10.

29. Cloarec, O.; Dumas, M. E.; Craig, A.; Barton, R. H.; Trygg, J.; Hudson, J.; Blancher, C.; Gauguier, D.; Lindon, J. C.; Holmes, E.; Nicholson, J., Statistical total correlation spectroscopy: An exploratory approach for latent biomarker identification from metabolic H-1 NMR data sets. Analytical chemistry 2005, 77, 1282-1289.

30. Posma, J. M.; Garcia-Perez, I.; De Iorio, M.; Lindon, J. C.; Elliott, P.; Holmes, E.; Ebbels, T. M.; Nicholson, J. K., Subset optimization by reference matching (STORM): an optimized statistical approach for recovery of metabolic biomarker structural information from $1 \mathrm{H}$ NMR spectra of biofluids. Analytical chemistry 2012, 84, 10694-701.

31. Hao, J.; Astle, W.; De lorio, M.; Ebbels, T. M., BATMAN--an R package for the automated quantification of metabolites from nuclear magnetic resonance spectra using a Bayesian model. Bioinformatics 2012, 28, 2088-90.

32. Ribéreau-Gayon, P.; Glories, Y.; Maujean, A.; Dubourdieu, D., Organic Acids in Wine. In Handbook of Enology, 2 ed.; John Wiley \& Sons, Ltd: 2006; Vol. 2, pp 1-49.

33. Velioglu, Y. S., Food acids: organic acids, volatile organic acids, and phenolic acids. In Advances in Food Biochemistry, Yildiz, F., Ed. CRC Press: Boca Raton, 2009; p 522.

34. Regueiro, J.; Vallverdu-Queralt, A.; Simal-Gandara, J.; Estruch, R.; Lamuela-Raventos, R. M., Urinary tartaric acid as a potential biomarker for the dietary assessment of moderate wine consumption: a randomised controlled trial. Brit J Nutr 2014, 111, 1680-1685.

35. Son, H. S.; Hwang, G. S.; Kim, K. M.; Ahn, H. J.; Park, W. M.; Van Den Berg, F.; Hong, Y. S.; Lee, C. H., Metabolomic Studies on Geographical Grapes and Their Wines Using H-1 NMR Analysis Coupled with Multivariate Statistics. J Agr Food Chem 2009, 57, 1481-1490.

36. Finkle, P., The fate of tartaric acid in the human body. J Biol Chem 1933, 100, 349-355.

37. Gry, J.; Larsen, J. C., Metabolism of L(+)-and D(-)-tartaric acids in different animal species. Archives of toxicology. Supplement. $=$ Archiv fur Toxikologie. Supplement 1978, 351-3.

38. Rebstockova, M.; Bartusek, M., Reactions of Boric-Acid with Oxalic, Glycolic and Tartaric-Acids. Collect Czech Chem C 1977, 42, 627-636. 
586 39. Mennen, L. I.; Sapinho, D.; Ito, H.; Bertrais, S.; Galan, P.; Hercberg, S.; Scalbert, A., Urinary 587 flavonoids and phenolic acids as biomarkers of intake for polyphenol-rich foods. Brit J Nutr 2006, 96, 588 191-198.

589 40. Zamora-Ros, R.; Urpi-Sarda, M.; Lamuela-Raventos, R. M.; Estruch, R.; Vazquez-Agell, M.; 590 Serrano-Martinez, M.; Jaeger, W.; Andres-Lacueva, C., Diagnostic performance of urinary resveratrol 591 metabolites as a biomarker of moderate wine consumption. Clin Chem 2006, 52, 1373-1380.

592 41. Zamora-Ros, R.; Urpi-Sarda, M.; Lamuela-Raventos, R. M.; Estruch, R.; Martinez-Gonzalez, M. 593 A.; Bullo, M.; Aros, F.; Cherubini, A.; Andres-Lacueva, C., Resveratrol metabolites in urine as a 594 biomarker of wine intake in free-living subjects: The PREDIMED Study. Free Radical Bio Med 2009, 46, 595 1562-1566.

$59642 . \quad$ Lord, R. S.; Burdette, C. K.; Bralley, J. A., Significance of urinary tartaric acid. Clin Chem 2005, $597 \quad 51,672-3$.

598 


\section{FIGURE CAPTIONS}

Figure 1: Study design for the controlled dietary intervention study $(n=19)$. The study is conducted against the background of a standardized diet where incremental amounts of grapes were provided as an afternoon snack to the volunteers.

Figure 2: A) Urinary excretion kinetics of tartaric acid in 6 volunteers after consumption of $50 \mathrm{~g}, 100 \mathrm{~g}$ and $200 \mathrm{~g}$ of grapes. The spectral region corresponding to the tartaric acid singlet at $\delta 4.34$ was absent in baseline samples (0h), visible in the $0-4 \mathrm{~h}$ samples (red) and 4-8h samples (green) after grape intake, and subsequently decreased towards baseline levels in the $8-12 \mathrm{~h}$ samples (blue) and 12-24h samples (magenta) after grape intake. B) 3D-PCA scores plot of spectra obtained from different varieties of red grapes with different geographic origins. Variance explained $\left(R^{2} x\right)$ for principal components (PC) 1,2 and 3 is $30 \%, 13 \%$ and $11 \%$, respectively. C) 3D-PCA scores plot of red grapes (red) vs green grapes (green) from different countries. $R^{2} x$ for principal components (PC) 1,2 and 3 is $25 \%, 13 \%$ and $11 \%$, respectively. Key, origin: $\succsim$ Peru, $\square$ Namibia, $\circ$ Chile, $\diamond$ India, $\nabla$ South Africa; variety: Ralli, Magenta, Jack's Salute, Black Princess, Pink Muscat, Red Globe, Sharad, Flame, Crimson.

Figure 3: A) Kernel density estimate (KDE) of the predicted PLS-DA scores shows good separation between red (red cross, $\times$ ) and green (green circle, $\circ$ ) grapes. $A n R^{2} Y$ of 0.65 shows the goodness of fit of the model and $a Q^{2} Y$ of 0.52 shows good capability for prediction. B) PLS-DA scores plot. C) PLS-DA loadings plot. The ${ }^{1} \mathrm{H}$ NMR peaks of 3 metabolites (phenylalanine, leucine and ethyl glucuronide) significantly different between red and green grapes are shown in individual panels, as 
is tartaric acid for which no significant difference in the concentration was found between red and green grapes.

Figure 4: Calibration curves of tartaric acid (mMol) excreted in urine after the intake of $0,50,100$ and $150 \mathrm{~g}$ of grapes in A) the first spot urine samples $2 \mathrm{~h}$ after grape intake, B) cumulative urine sample from after lunch to before dinner (CS2), C) cumulative urine sample from after dinner to next day fasting urine sample (CS3) and D) $24 \mathrm{~h}$ urine samples. The shaded area represents the $95 \%$ confidence interval obtained using bootstrap resampling.

Figure 5: Bland-Altman plots of the actual and estimated grape intake in A) CS2 samples, B) CS3 samples and C) $24 \mathrm{~h}$ urine samples, showing that the predictions of amount of grape intake in grams is close to the real intake of 50,100 and $150 \mathrm{~g}$ of grapes. The green line indicates mean \pm 1 S.D., blue \pm 2 S.D. and red \pm 3 S.D. 
Figure 1:

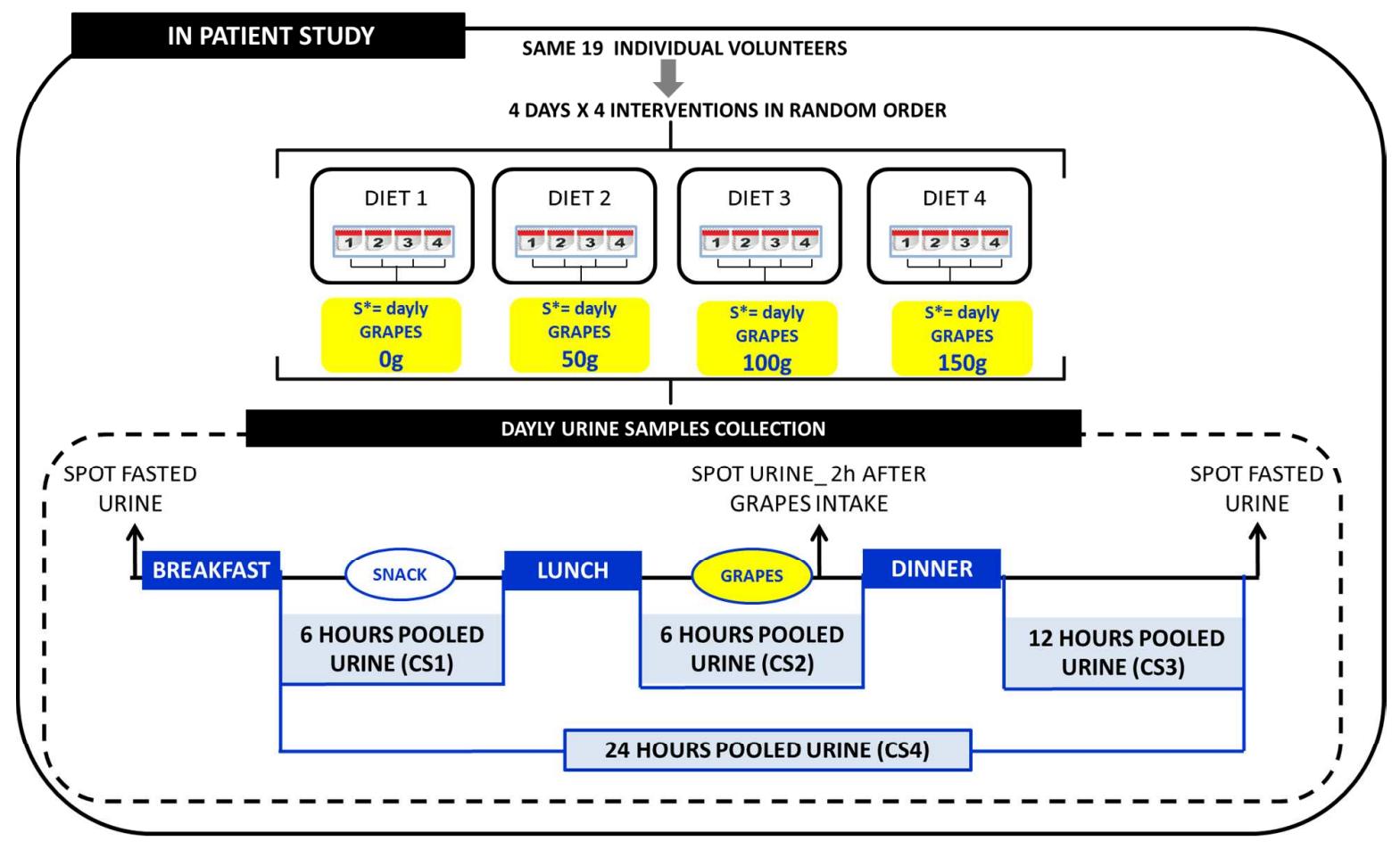


Figure 2:
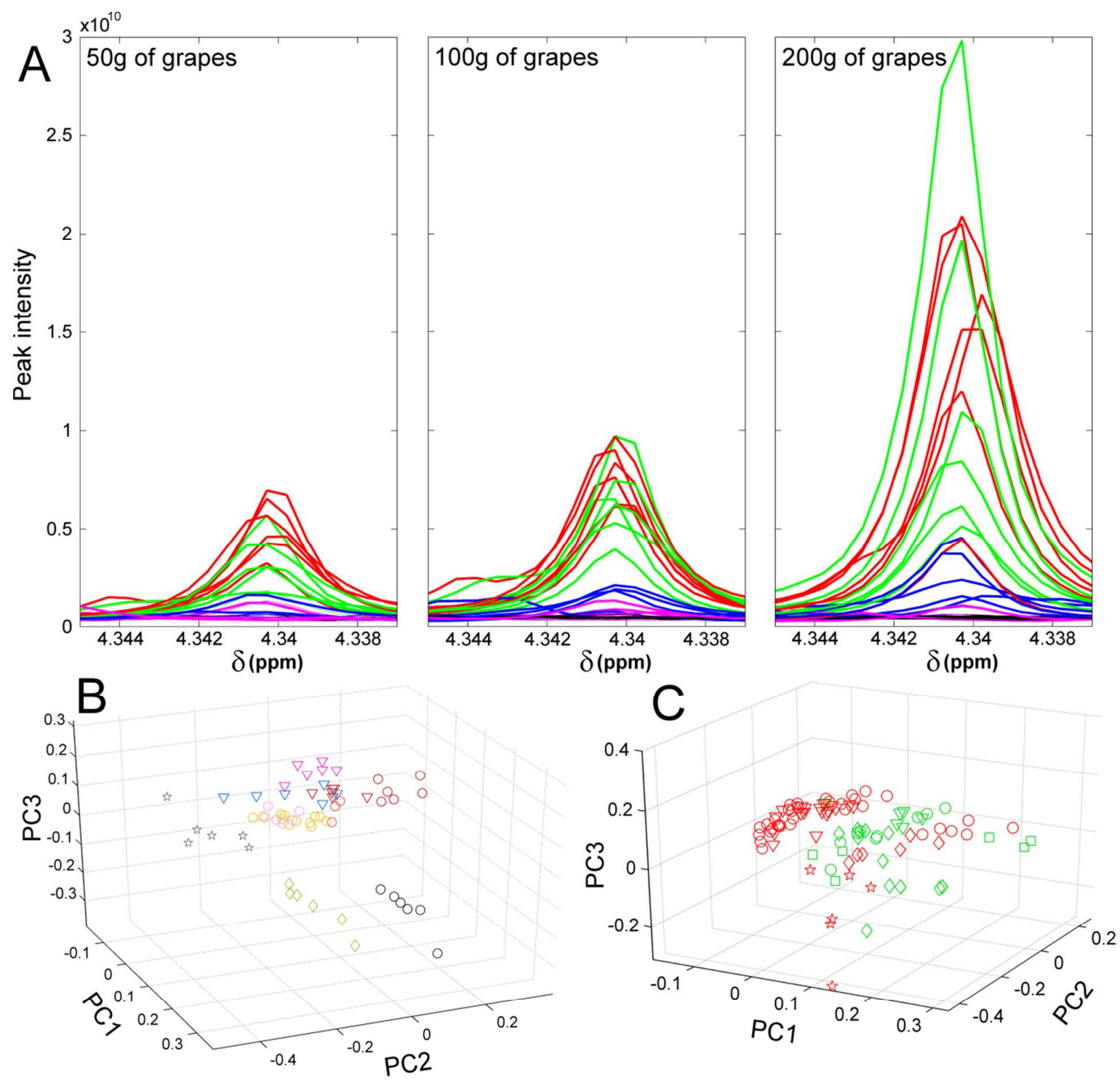
Figure 3:
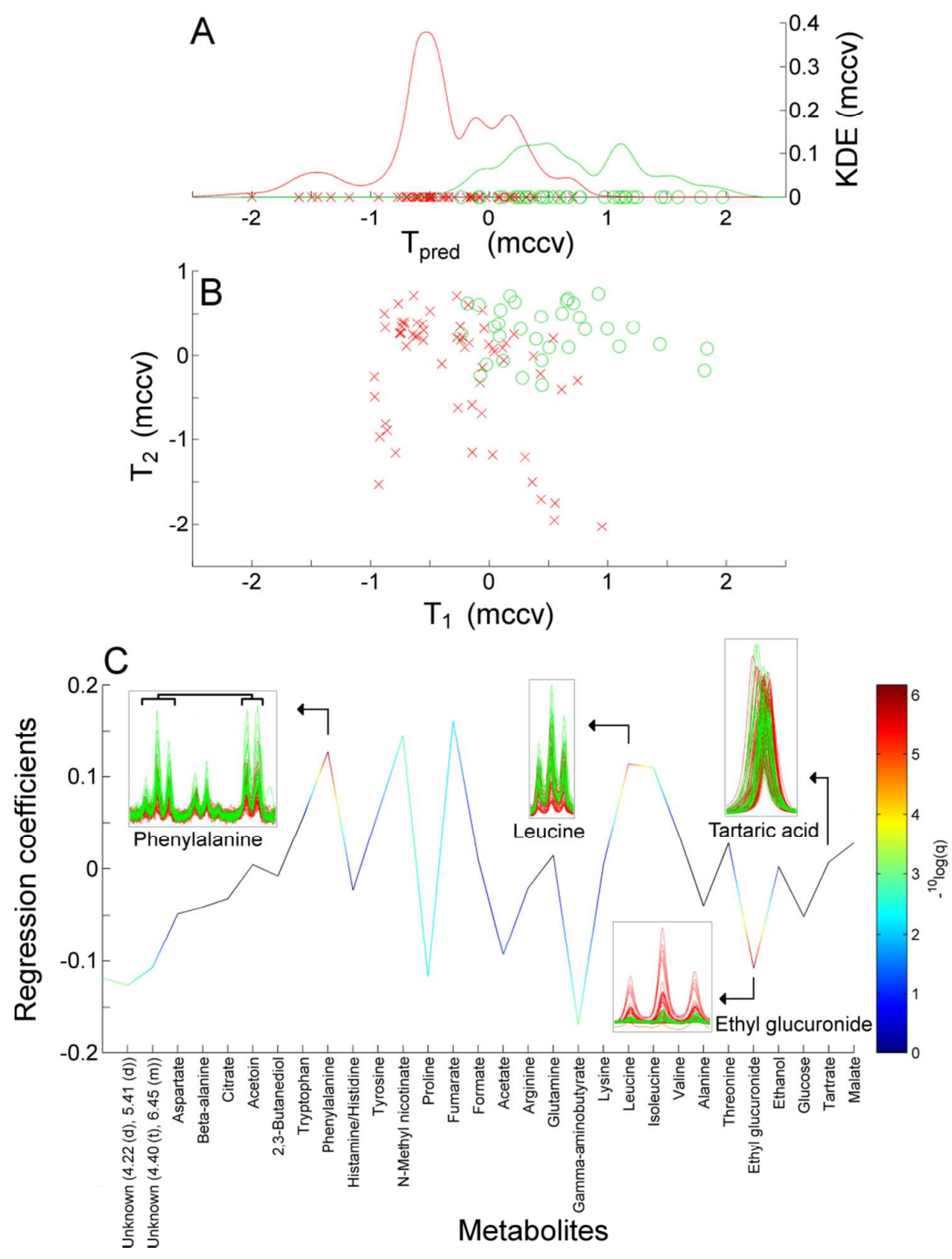
Figure 4:
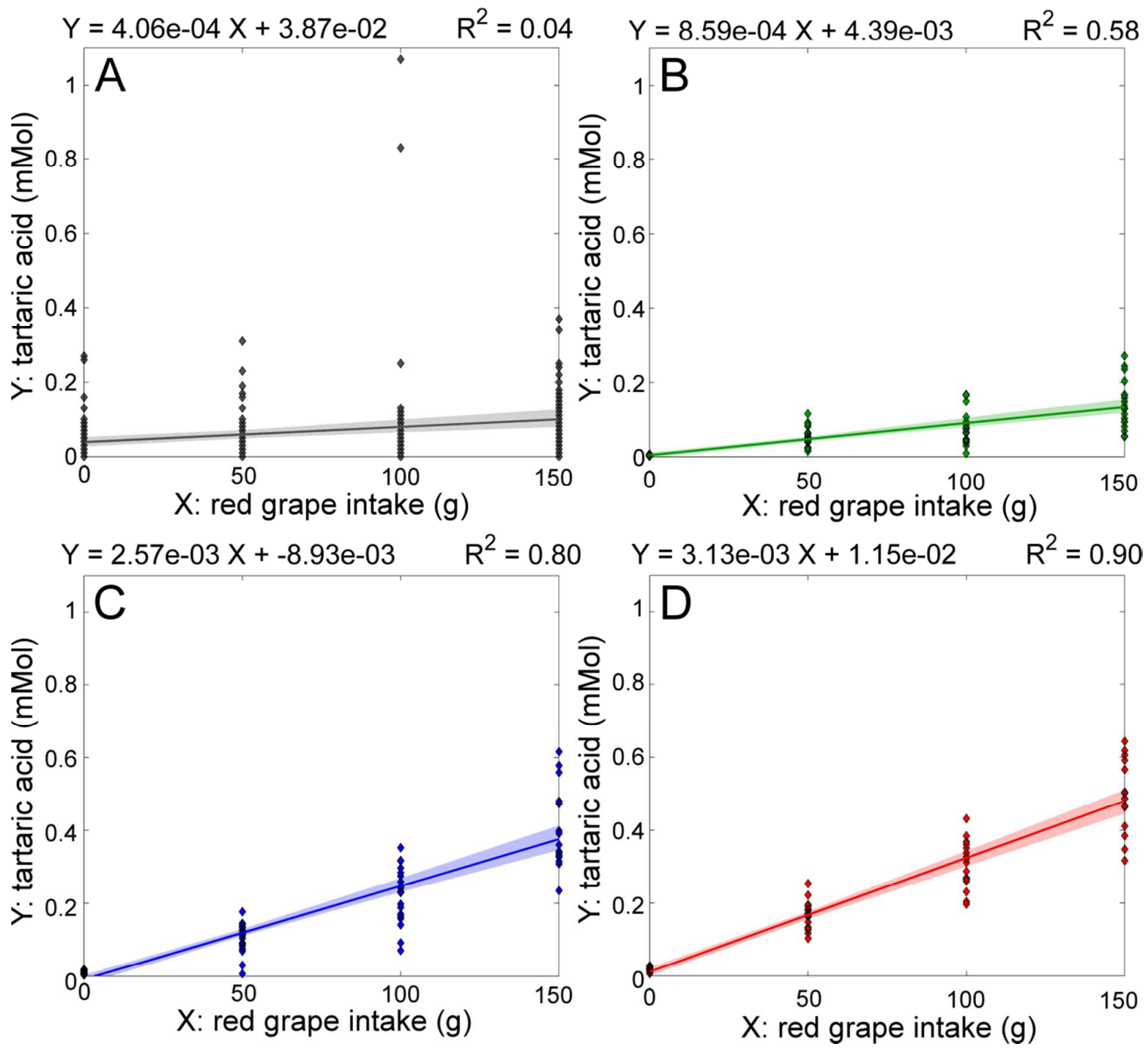
Figure 5:
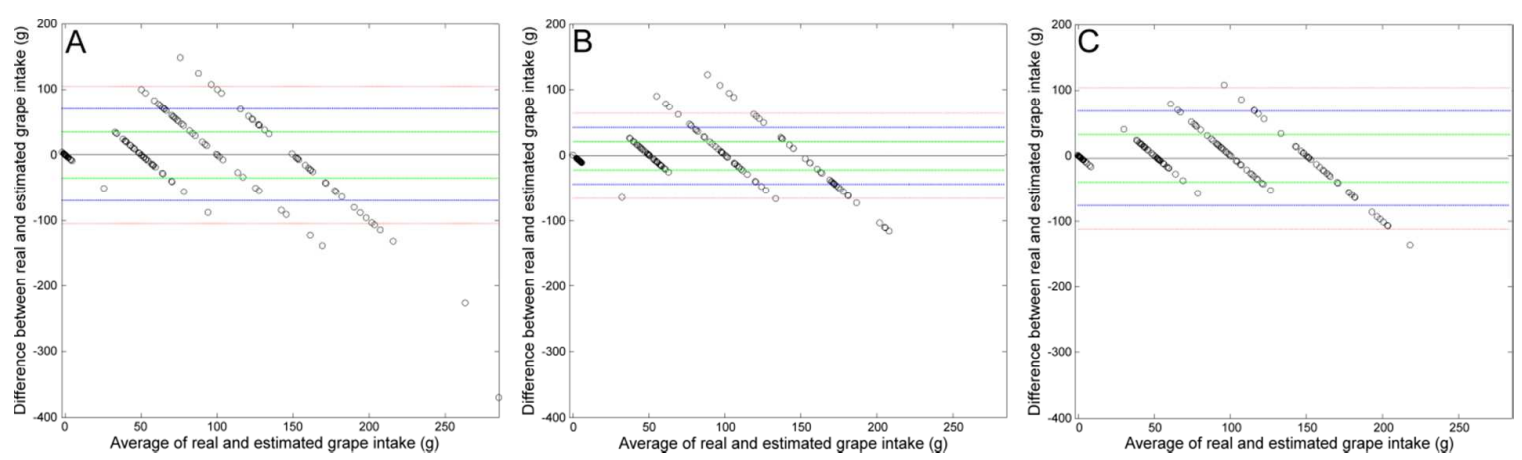

600 
TOC Graphic:
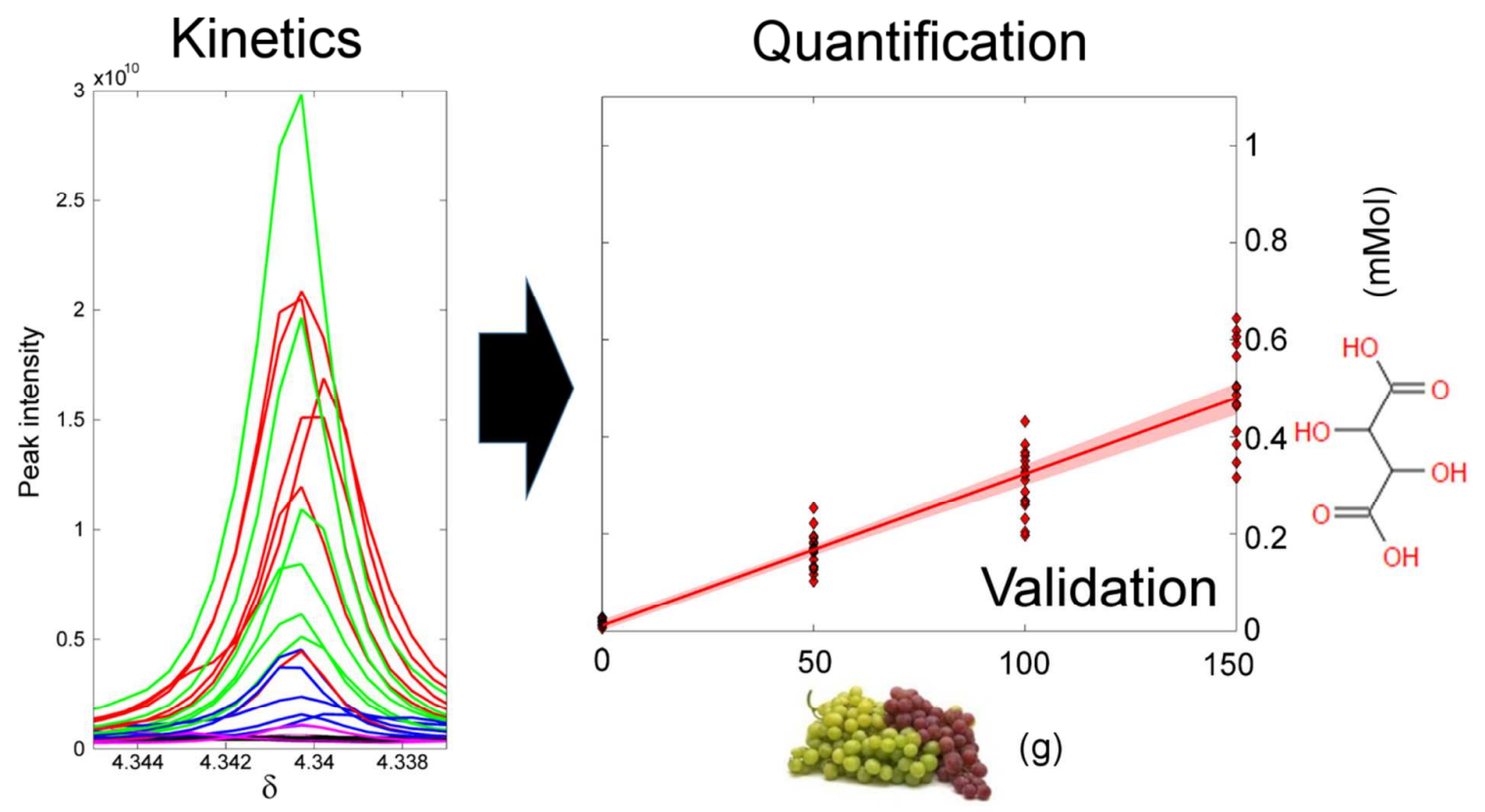